Check for updates

Cite this: RSC Adv., 2017, 7, 53532

\title{
Bio-conjugation of graphene quantum dots for targeting imaging $\dagger$
}

\author{
Fei Jia, (D) * Shuyu Lv and Sha Xu
}

Graphene quantum dots (GQDs) with strong photoluminescence (PL) and chemical modification have attracted tremendous interest for fundamental and applied research owing to their special low-cost production and low toxicity. However, most of the research focused on the GQDs without much targeted design, which limited their applications in specific bio-based fields. Inspired by this fascinating conundrum, we demonstrate GQD-based bio-conjugation. Relying on sedimentation coefficient differences for the density gradient ultracentrifuge (DGU) separation of conjugates with different proteins, ultra-purified conjugates were obtained. We demonstrate the first targeted imaging in both cells and tissue models with single or multi color staining, which shows universality for different kinds of biomolecules.

Received 31st October 2017

Accepted 15th November 2017

DOI: 10.1039/c7ra11963a

rsc.li/rsc-advances

Fluorescent materials play an important role in investigating biological issues as a "visible" marker. ${ }^{1,2}$ In the evolution of fluorescent materials, increasing efforts have been devoted to the development of heavy-metal-free and low-toxicity photoluminescence (PL) nano-probes. Carbon dots (CDs: including graphene quantum dot (GQDs), ${ }^{3-7}$ carbon nanodots (CNDs), ${ }^{8-11}$ polymer dots (PDs) ${ }^{12-15}$ and polymer carbon dots (PCDs) ${ }^{16-20}$ ), one class of these nano-probes, have drawn much attention during recent years. ${ }^{10,21}$ GQDs possess high photo-stability, tunable emission, chemical inertness, good biocompatibility, low toxicity, photoelectric/optical properties ${ }^{22-26}$ and convenient surface modification, and can be achieved by low-cost production. Therefore, GQDs could find use in biomedical applications, such as bioimaging, ${ }^{27,28}$ drug delivery, ${ }^{29,30}$ thermal therapy, ${ }^{31,32}$ DNA cleavage, ${ }^{33}$ sensors,${ }^{34}$ etc.

Bioimaging is an emerging research field aimed at using sophisticated bioimaging probes to visualize specific molecular pathways in vivo or in vitro. ${ }^{35,36}$ Currently, the majority of bioimaging probes utilized in clinical practice are small-molecule compounds that tend to be unstable, toxic, nonspecific, and rapidly cleared. GQDs have the potential to be remarkably successful in the field of bioimaging due in part to their excellent optical properties and extremely low cytotoxicity, but also due to their suitable size and surface chemistry. ${ }^{37,38}$ The size of GQDs is always $c a$. $3-10 \mathrm{~nm}$ and lots of oxygen or amino based groups, which are beneficial for bio-labeling process (e.g. endocytose), are found on the surface of GQDs. ${ }^{7,39}$ However, there are few work focusing on the targeting imaging through

School of Chemical Engineering, Changchun University of Technology, Changchun, 130012, China. E-mail: jiafei19861112@163.com

$\dagger$ Electronic supplementary information (ESI) available: Characterization of GQDs, more cell imaging and in vivo data. See DOI: 10.1039/c7ra11963a the bio-conjugation of GQDs. It is very important to use the targeting GQDs for specific imaging position, which can afford efficient imaging speed, high signal-to-noise ratio (SNR) as well as multi-colors issue imaging: ${ }^{40-42}$

Although there are kinds of bio-conjugation methods developing with high reaction activity, all the reaction mixture generally contains unbound fluorophores (small molecules or nanomaterials), which cause the background problem in the staining process. ${ }^{43-45}$ Therefore, the efficient separation of fluorophore bioconjugates from unbound fluorophore is necessary. The density gradient ultracentrifuge (DGU) is a reliable and flexible approach to purify these kinds of conjugates. DGU works by exploiting subtle differences in buoyant density. The species of interest are loaded into an aqueous solution with a known density gradient. Under the centripetal force of an ultracentrifuge, the species sediment toward their respective isopycnic points (i.e. the position where their density matches that of the gradient). ${ }^{46} \mathrm{As}$ a result, it is very important to purify the GQDs bio-conjugation for further targeting staining.

Herein, graphene quantum dots with green PL emission and carboxyl groups were used for the bio-conjugation with amino groups of proteins, and the conjugates of streptavidin (SA) @GQDs, anti-mouse@GQDs and Erbitux@GQDs were successfully prepared and purified by the density gradient ultracentrifuge (DGU) route. The conjugates between GQDs and different proteins possess controllable targeting capacity to label the specific position of biological systems. For example, the anti mouse@GQDs/mouse anti-Her 2 was used to stain SKOV-3 (human ovarian carcinoma) cell; cluster of differentiation 31 (CD 31)@biotin and SA@GQDs were used to stain vessel; Erbitux@GQDs were used to stain squamous cell carcinoma (SCC) cell. 
GQDs were prepared by previously reported "nano-cutting" and solvothermal method from graphite powder and $\mathrm{HNO}_{3} /$ $\mathrm{H}_{2} \mathrm{SO}_{4}$, developed by Yang group, ${ }^{47,48}$ as shown in Scheme S1. $\dagger$ The average diameter of the prepared GQDs was $c a .4 .5 \mathrm{~nm}$ with crystal lattice $c a$. $0.21 \mathrm{~nm}$ (Fig. 1a and S2 $\dagger$ ). The MALDI-TOF MS spectra of GQDs showed the molecular weights were mainly between 1250-2750 with an regular interval (58 Da). It indicated that the neighbouring GQDs differed by " $\mathrm{C}_{2} \mathrm{O}_{2} \mathrm{H}_{2}$ " groups (Fig. 1b), and the actual molecule weight will be larger than this value because big GQDs are hard to be ionized. In the FTIR analysis of GQDs, stretching vibrations of $\mathrm{C}-\mathrm{OH}$ at $3430 \mathrm{~cm}^{-1}$, and a vibrational absorption band of $\mathrm{C}=\mathrm{O}$ at $1635 \mathrm{~cm}^{-1}$ were all observed (Fig. 1c). ${ }^{\mathbf{8} 47}$ In addition, surface groups were also investigated by XPS analysis (Fig. S3 $\dagger$ ). The $-\mathrm{OH}$ was mainly produced by the cutting position through epoxy, while the - $\mathrm{COOH}$ and carbonyl was the stable oxidation state. The carboxyl groups were further used for bio-conjugation. GQDs possess optimal excitation and emission wavelengths at $c a$. $468 \mathrm{~nm}$ and $525 \mathrm{~nm}$, respectively. We have tested the absolute quantum yield of present GQDs, and the value is $4.7 \%$. The PL center of GQDs was in surface state which was induced by the hybridization structure of edge groups and connected carbon core, and the participant edge groups for green emission mainly resulted from carboxyl and amide. ${ }^{49,50}$

The specific binding used in this work was based on the bioconjugation, e.g., streptavidin (SA) and biotin. ${ }^{51}$ First of all, the covalent SA@GQDs and bovine serum albumin (BSA)@biotin were prepared by standard 1-ethyl-3-(3-dimethylaminopropyl) carbodiimide hydrochloride (EDC) method and biotinylated reagent, respectively (EDC was used to react with the carboxyl groups on the surface of GQDs, forming an unstable reactive $O$-acylisourea intermediate. Subsequently, $N$-hydroxysulfosuccinimide (Sulfo-NHS) was added to convert it to a semistable amine-reactive NHS-ester. ${ }^{52,53}$ The amine groups of proteins then covalently conjugated with the NHS-ester modified GQDs to produce protein@GQDs). ${ }^{51}$ For purifying the BSA@biotin, due to the biotinylated molecules are relatively small, the centrifuge filter with molecule weight $30 \mathrm{kDa}$ can be
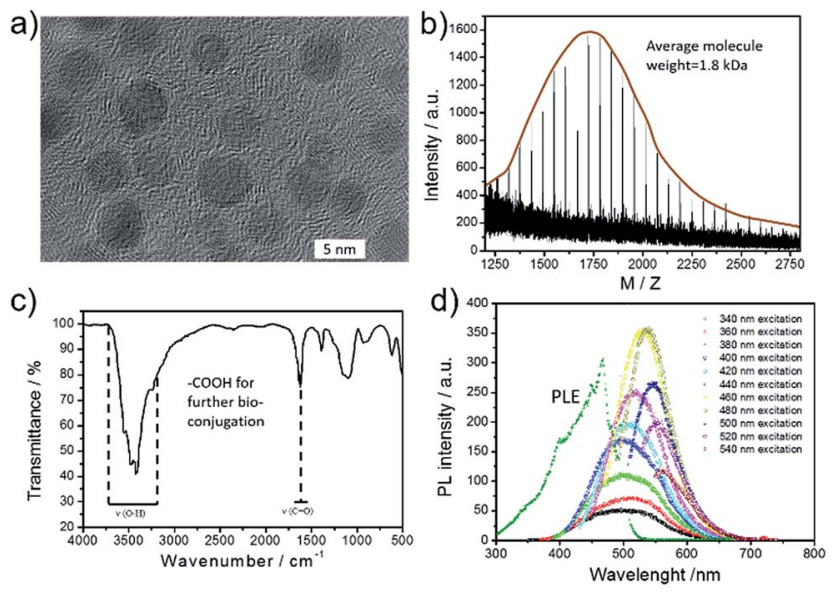

Fig. 1 The used GQDs for bio-conjugation. (a) TEM image of GQDs (b) MALDI-TOF MS spectrum of GQDs. (c) FT-IR spectrum of GQDs. (d) The excitation and emission spectra of GQDs. used to remove the un-conjugated biotin. For purifying the SA@GQDs, the density gradient ultracentrifuge (DGU) was applied to separate the SA@GQDs from the mixtures of free SA, free GQDs. The used gradient sucrose was from 15 to $50 \%$ (5\% interval, the final column will be a homogeneous linear gradient after tilting $20^{\circ}$ for 45 minutes), then we tested the DGU column after ultracentrifuge (Fig. 2a-c), and the desired SA@GQDs conjugates were concentrated at the gradient positions of 20$25 \%$ (Fig. 2b). On the top of the fluorescent column section, there are free GQDs and SA; in the middle of the fluorescent section, there should be the desired conjugates between single SA and GQDs, or few SA and GQDs; at the bottom of the fluorescent section, there are possible the multimer with several SA and GQDs conjugates.

To achieve multiple targeting tissue staining with the bioconjugates, GQDs were further covalently bonded to another two typical proteins (anti-mouse secondary antibodies and Erbitux). Similar DGU purification was used to obtain the selected conjugation with high purifications. For testing the conjugation between GQDs and different proteins, the Bolt 4$12 \%$ Bis-Tris gel electrophoresis was used to confirm the conjugate formation of proteins and GQDs via fluorescence imaging, since GQDs have strong fluorescence signals under blue light illumination. As shown in Fig. 2d, SA@GQDs possess three PL bands between $50 \mathrm{kDa}$ and $70 \mathrm{kDa}$, resulting from the dimer and trimer conjugates; anti-mouse@GQDs show the molecule weight at 115-140 kDa; for Erbitux@GQDs, there is a strong band at $150 \mathrm{kDa}$, and also two weak bands at larger molecule section, which are due to the dimer and trimer conjugations. Fortunately, the multimer of the protein, which is also powerful for targeting conjugations, don't affect the
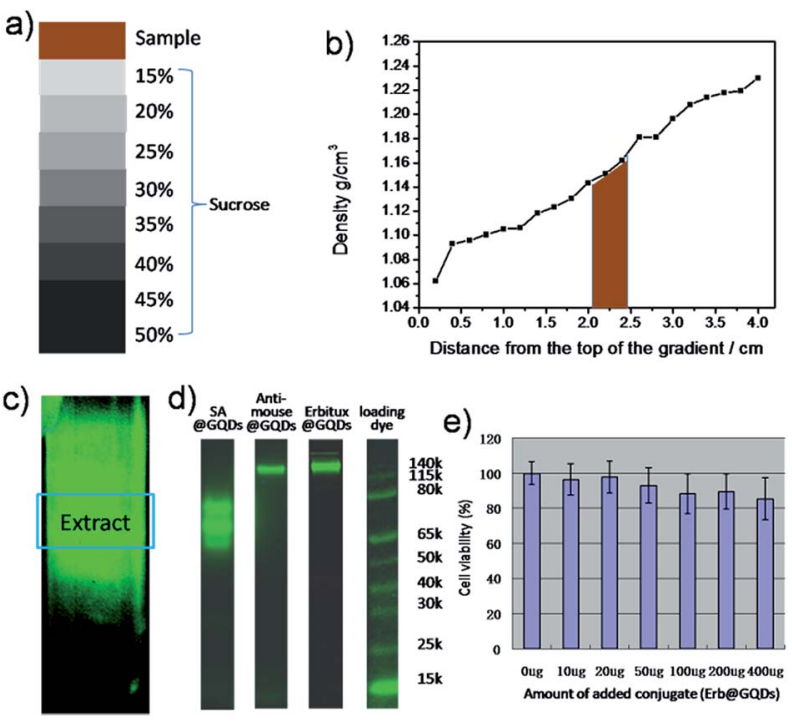

Fig. 2 (a) The sucrose DGU gradient used for purifying the conjugates. (b) The desired SA@GQDs positions in the DGU column. (c) PL image of the DGU column after ultracentrifuge. (d) Bolt 4-12\% Bis-Tris gel electrophoresis analysis of SA@GQDs, anti-mouseaGQDs and Erbitux@GQDs, respectively. The loading dye with confirmed molecule weight was tested as references. (e) The cell viability of conjugate Erbitux@GQDs (SCC cell). 
biological activity. ${ }^{\mathbf{5 4 , 5 5}}$ More importantly, the GQDs show very cell toxicity which is ideal for in vivo staining.

To test the performance of the targeting system, first of all, the targeting cell imaging was tested by SKOV-3 cell model. The cultured SKOV-3 stained by anti-mouse@GQDs/mouse anti-Her 2 and $4^{\prime}$,6-diamidino-2-phenylindole dihydrochloride (Dapi). ${ }^{56}$ There are Her 2 proteins receptors located on the surface of SKOV-3 cell membrane, which can be conjugated by mouse anti-Her 2 followed by anti-mouse@GQDs. From bright field image of Fig. 3a, the cells kept the normal morphology, and the blue fluorescence (Dapi channel) can be seen in the nucleus while the green fluorescence (GFP channel) was located on the cell membrane of SKOV-3. The overlay images (Fig. 3a and S4†) indicated perfect staining of the nucleus and Her 2 proteins receptors of cell membrane, respectively. Furthermore, due to the universality of the conjugation with bio-molecules, other targeting staining could be achieved.

The targeting imaging provides the possibility to stain different positions of the tissue or living body at the same time. We tested the multi-color imaging by using the SKOV 3 tumor tissue as a model case. The Dapi with blue emission was used for staining the nucleus; the anti mouse@IR-680 and mouse anti-Her 2 were used to stain the SKOV 3 cells of the tumor; the anti CD31@biotin and SA@GQDs were used to stain the vessels of the tumor. Fig. 3b shows the three color imaging of the SKOV 3 tumor. The blue nucleus was encircled by the red cell membrane, and the green vessel can also be recognized spreading the whole tumor slide.

The GQDs can also be used for monoclonal antibody conjugation, which will be "one-step" binding to the cell or organ efficiently. ${ }^{57}$ Erbitux was used as a proof-of-concept model. Erbitux (cetuximab) is a recombinant, human/mouse chimeric monoclonal antibody that binds specifically to the extracellular domain of the EGFR. The Erbitux@GQDs conjugation possesses a molecule weight slightly more than $150 \mathrm{kDa}$, and bright fluorescence (Fig. 2d). Fig. 4a shows the scheme of biological fixation between the Erbitux@GQDs and EGFR of the

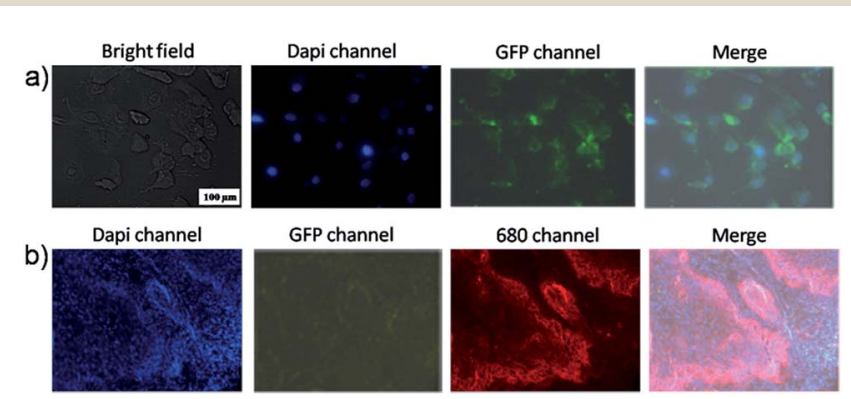

Fig. 3 Targeting cell imaging and tissue staining of proteinaGQDs. (a) SKOV 3 cell imaging by mouse anti-Her 2 and anti-mouse@GQDs, and the nucleus was stained by Dapi for location. (b) SKOV 3 tumor imaging: the vessel staining by anti CD31abiotin and SACGQDs; the cell membrane was by mouse anti-Her 2 and anti-mouseaIR-680; the nucleus was also stained by Dapi. For GFP channel of GQDs, due to the low quantum yield, we have to tune the contrast/brightness to reach the same quality compared with the Dapi and 680 (IR-680 dyes) channels. The emission of Dapi also has some signal in GQDs channel, and we have re-draw the data to get rid of the Dapi signal.

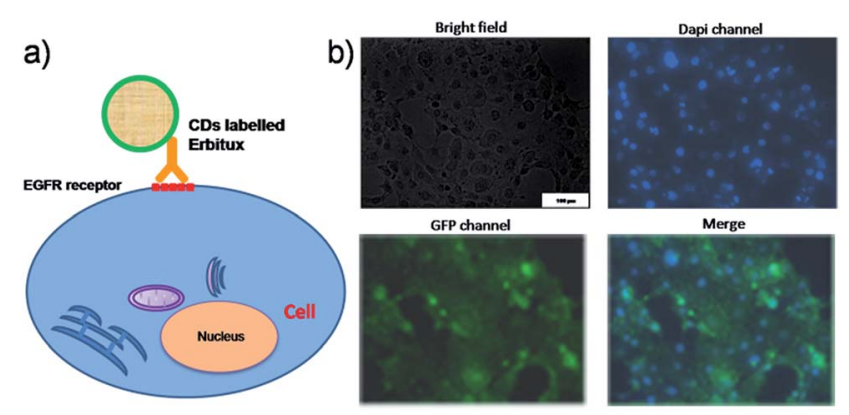

Fig. 4 (a) SCC cell imaging by Erbitux@GQDs which is binding to the EGFR receptor of SCC cell membrane. (b) The bright field, and PL picture at different channels. After merging the PL picture of Dapi and GFP channels, the successful staining of membrane and nucleus was observed. The quantum yield of GQDs is much lower than Dapi, and we have to tune the contrast/brightness to reach the same quality between Dapi and GQDs. The emission of Dapi also has some signal in GQDs channel, and we have re-draw the data to get rid of the Dapi signal.

SCC cells. After suitable cultivating, the targeted imaging could be observed on the membrane of the SCC cells (Fig. $4 \mathrm{~b}$ and S5 $\dagger$ ). The bright field, Dapi staining of the nucleus and the merger imaging indicated the perfect staining of the EGFR of the SCC cells. The in vivo targeting imaging was also conducted on SCC tumor model (Fig. S6†), showing great tumor targeting capacity also with the non-specific liver uptake. H\&E staining study of GQDs@conjugate was added in Fig. S7, $\dagger$ and it show very low in vivo toxicity of the present conjugate.

\section{Conclusions}

In conclusion, high-performance GQDs based bio-conjugates were developed. Relying on sedimentation coefficient differences for the density gradient ultracentrifuge (DGU) separation of conjugate with different proteins, ultra-purified conjugates were obtained. By using gel electrophoresis to quickly screen conjugation efficiency, we demonstrate that DGU separated conjugates display the highest selectivity out of any carbon based imaging agent to date. At last, the targeting cell imaging and tissue staining were achieved by the conjugates of SA@GQDs, Erbitux@GQDs and anti mouse@GQDs with very high efficiency, demonstrating the first histological imaging with GQDs based conjugates. The present work endows the fluorescent carbon based materials with promising targeting property, which is of great significance to practical bio-based applications.

\section{Conflicts of interest}

There are no conflicts to declare.

\section{Acknowledgements}

This work was supported by the NSFC 20160323MA1. 


\section{Notes and references}

1 C. Vinegoni, I. Botnaru, E. Aikawa, M. A. Calfon, Y. Iwamoto, E. J. Folco, V. Ntziachristos, R. Weissleder, P. Libby and F. A. Jaffer, Sci. Transl. Med., 2011, 3, 84ra45.

2 H. Zhang, Y. Liu, D. Yao and B. Yang, Chem. Soc. Rev., 2012, 41, 6066-6088.

3 Y. Li, Y. Hu, Y. Zhao, G. Shi, L. Deng, Y. Hou and L. Qu, Adv. Mater., 2011, 23, 776-780.

4 S. Zhu, J. Zhang, C. Qiao, S. Tang, Y. Li, W. Yuan, B. Li, L. Tian, F. Liu, R. Hu, H. Gao, H. Wei, H. Zhang, H. Sun and B. Yang, Chem. Commun., 2011, 47, 6858-6860.

5 S. Zhu, J. Zhang, X. Liu, B. Li, X. Wang, S. Tang, Q. Meng, Y. Li, C. Shi, R. Hu and B. Yang, RSC Adv., 2012, 2, 2717.

6 S. Zhu, X. Zhao, Y. Song, J. Zhang and B. Yang, Graphene Science Handbook, 2016, vol. 10, p. 163.

7 S. Zhu, S. Tang, J. Zhang and B. Yang, Chem. Commun., 2012, 48, 4527-4539.

8 B. Kong, A. Zhu, C. Ding, X. Zhao, B. Li and Y. Tian, Adv. Mater., 2012, 24, 5844-5848.

9 S. Zhu, X. Zhao, Y. Song, S. Lu and B. Yang, Nano Today, 2016, 11, 128-132.

10 Y. Song, S. Zhu and B. Yang, RSC Adv., 2014, 4, 27184.

11 S. Zhu, Q. Meng, L. Wang, J. Zhang, Y. Song, H. Jin, K. Zhang, H. Sun, H. Wang and B. Yang, Angew. Chem., Int. Ed., 2013, 52, 3953-3957.

12 S. Zhu, J. Zhang, L. Wang, Y. Song, G. Zhang, H. Wang and B. Yang, Chem. Commun., 2012, 48, 10889-10891.

13 S. Zhu, L. Wang, N. Zhou, X. Zhao, Y. Song, S. Maharjan, J. Zhang, L. Lu, H. Wang and B. Yang, Chem. Commun., 2014, 50, 13845-13848.

14 S. Zhu, Y. Song, J. Shao, X. Zhao and B. Yang, Angew. Chem., Int. Ed., 2015, 54, 14626-14637.

15 S. Zhu, J. Zhang, Y. Song, G. Zhang, H. Zhang and B. Yang, Acta Chim. Sin., 2012, 70, 2311.

16 J. Shao, S. Zhu, H. Liu, Y. Song, S. Tao and B. Yang, Adv. Sci., 2017, 1700395.

17 Y. Song, S. Zhu, J. Shao and B. Yang, J. Polym. Sci., Part A: Polym. Chem., 2017, 55, 610-615.

18 S. Tao, S. Zhu, T. Feng, C. Xia, Y. Song and B. Yang, Mater. Today Chem., 2017, 6, 13-25.

19 S. Tao, Y. Song, S. Zhu, J. Shao and B. Yang, Polymer, 2017, 116, $472-478$.

20 S. Lu, L. Sui, J. Liu, S. Zhu, A. Chen, M. Jin and B. Yang, Adv. Mater., 2017, 29, 1603443.

21 S. Zhu, Y. Song, X. Zhao, J. Shao, J. Zhang and B. Yang, Nano Res., 2015, 8, 355-381.

22 S. Y. Lim, W. Shen and Z. Gao, Chem. Soc. Rev., 2015, 44, 362-381.

23 C. Yu, T. Xuan, Y. Chen, Z. Zhao, X. Liu, G. Lian and H. Li, J. Alloys Compd., 2016, 688, 611-619.

24 C. Yu, T. Xuan, D. Yan, S. Lou, X. Hou, Y. Chen, J. Wang and H. Li, Sens. Actuators, B, 2017, 253, 900-910.

25 Q. Qu, A. Zhu, X. Shao, G. Shi and Y. Tian, Chem. Commun., 2012, 48, 5473-5475.

26 X. Li, S. Zhu, B. Xu, K. Ma, J. Zhang, B. Yang and W. Tian, Nanoscale, 2013, 5, 7776-7779.
27 S. Zhu, J. Zhang, S. Tang, C. Qiao, L. Wang, H. Wang, X. Liu, B. Li, Y. Li, W. Yu, X. Wang, H. Sun and B. Yang, Adv. Funct. Mater., 2012, 22, 4732-4740.

28 L. Wang, Y. Wang, T. Xu, H. Liao, C. Yao, Y. Liu, Z. Li, Z. Chen, D. Pan, L. Sun and M. Wu, Nat. Commun., 2014, 5, 5357.

29 L. Lin, M. Rong, F. Luo, D. Chen, Y. Wang and X. Chen, TrAC, Trends Anal. Chem., 2014, 54, 83-102.

30 L. Li, G. Wu, G. Yang, J. Peng, J. Zhao and J. J. Zhu, Nanoscale, 2013, 5, 4015-4039.

31 Z. M. Markovic, B. Z. Ristic, K. M. Arsikin, D. G. Klisic, L. M. Harhaji-Trajkovic, B. M. Todorovic-Markovic, D. P. Kepic, T. K. Kravic-Stevovic, S. P. Jovanovic, M. M. Milenkovic, D. D. Milivojevic, V. Z. Bumbasirevic, M. D. Dramicanin and V. S. Trajkovic, Biomaterials, 2012, 33, 7084-7092.

32 J. Ge, M. Lan, B. Zhou, W. Liu, L. Guo, H. Wang, Q. Jia, G. Niu, X. Huang, H. Zhou, X. Meng, P. Wang, C. S. Lee, W. Zhang and X. Han, Nat. Commun., 2014, 5, 4596.

33 X. Zhou, Y. Zhang, C. Wang, X. Wu, Y. Yang, B. Zheng, H. Wu, S. Guo and J. Zhang, ACS Nano, 2012, 6, 6592-6599. 34 Y. Mao, Y. Bao, L. Yan, G. Li, F. Li, D. Han, X. Zhang and L. Niu, RSC Adv., 2013, 3, 5475.

35 P. G. Luo, F. Yang, S.-T. Yang, S. K. Sonkar, L. Yang, J. J. Broglie, Y. Liu and Y.-P. Sun, RSC Adv., 2014, 4, 10791. 36 J. Zhang and S.-H. Yu, Mater. Today, 2016, 19, 382-393.

37 C. Wu, C. Wang, T. Han, X. Zhou, S. Guo and J. Zhang, Adv. Healthcare Mater., 2013, 2, 1613-1619.

38 M. Nurunnabi, Z. Khatun, K. M. Huh, S. Y. Park, D. Y. Lee, K. J. Cho and Y. K. Lee, ACS Nano, 2013, 7, 6858-6867.

39 J. Shen, Y. Zhu, X. Yang and C. Li, Chem. Commun., 2012, 48, 3686-3699.

40 A. Zhu, C. Ding and Y. Tian, Sci. Rep., 2013, 3, 2933.

41 Q. Liu, B. Guo, Z. Rao, B. Zhang and J. R. Gong, Nano Lett., 2013, 13, 2436-2441.

42 M. Zheng, S. Liu, J. Li, D. Qu, H. Zhao, X. Guan, X. Hu, Z. Xie, X. Jing and Z. Sun, Adv. Mater., 2014, 26, 3554-3560.

43 X. T. Zheng, H. L. He and C. M. Li, RSC Adv., 2013, 3, 24853. 44 X. Wang, X. Sun, J. Lao, H. He, T. Cheng, M. Wang, S. Wang and F. Huang, Colloids Surf., B, 2014, 122, 638-644.

45 P. Nigam, S. Waghmode, M. Louis, S. Wangnoo, P. Chavan and D. Sarkar, J. Mater. Chem. B, 2014, 2, 3190.

46 M. Grossi, M. Morgunova, S. Cheung, D. Scholz, E. Conroy, M. Terrile, A. Panarella, J. C. Simpson, W. M. Gallagher and D. F. O'Shea, Nat. Commun., 2016, 7, 10855.

47 S. Zhu, J. Shao, Y. Song, X. Zhao, J. Du, L. Wang, H. Wang, K. Zhang, J. Zhang and B. Yang, Nanoscale, 2015, 7, 79277933.

48 S. Zhu, N. Zhou, Z. Hao, S. Maharjan, X. Zhao, Y. Song, B. Sun, K. Zhang, J. Zhang, H. Sun, L. Lu and B. Yang, RSC Adv., 2015, 5, 39399-39403.

49 L. Wang, S. J. Zhu, H. Y. Wang, S. N. Qu, Y. L. Zhang, J. H. Zhang, Q. D. Chen, H. L. Xu, W. Han, B. Yang and H. B. Sun, ACS Nano, 2014, 8, 2541-2547.

50 S. Zhu, Y. Song, J. Wang, H. Wan, Y. Zhang, Y. Ning and B. Yang, Nano Today, 2017, 13, 10-14. 
51 M. Kano, A. E. Sosulski, L. Zhang, H. D. Saatcioglu, D. Wang, N. Nagykery, M. E. Sabatini, G. Gao, P. K. Donahoe and D. Pepin, Proc. Natl. Acad. Sci. U. S. A., 2017, 114, E1688E1697.

52 N. Zhou, Z. Hao, X. Zhao, S. Maharjan, S. Zhu, Y. Song, B. Yang and L. Lu, Nanoscale, 2015, 7, 15635-15642.

53 B. Sun, B. Zhao, D. Wang, Y. Wang, Q. Tang, S. Zhu, B. Yang and H. Sun, Nanoscale, 2016, 8, 9837-9841.

54 E. L. Rosenthal, J. M. Warram, E. de Boer, T. K. Chung, M. L. Korb, M. Brandwein-Gensler, T. V. Strong,
C. E. Schmalbach, A. B. Morlandt, G. Agarwal, Y. E. Hartman, W. R. Carroll, J. S. Richman, L. K. Clemons, L. M. Nabell and K. R. Zinn, Clin. Cancer Res., 2015, 21, 3658-3666.

55 S. Zhu, Y. Li, J. Zhang, C. Lu, X. Dai, F. Jia, H. Gao and B. Yang, J. Colloid Interface Sci., 2010, 344, 541-546.

56 H. S. Choi, S. L. Gibbs, J. H. Lee, S. H. Kim, Y. Ashitate, F. Liu, H. Hyun, G. Park, Y. Xie, S. Bae, M. Henary and J. V. Frangioni, Nat. Biotechnol., 2013, 31, 148-153.

57 C. Ding, A. Zhu and Y. Tian, Acc. Chem. Res., 2014, 47, 20-30. 